\title{
Reseña histórica de los colegios humanísticos costarricenses
}

\section{Historical Account of Humanistic Secondary Schools}

\author{
Evelyn Vargas Hernández \\ Ex-Directora Ejecutiva del CHC, Campus Omar Dengo, Heredia \\ evelyn.vargas.hernández@una.cr \\ Johnny Vásquez Lemitre \\ Director Ejecutivo del CHC, Campus Coto, UNA \\ johnny_vasquez66@yahoo.com
}

\section{Resumen}

El sistema de colegios humanísticos fomenta la formación humanista de una ciudadanía comprometida con el análisis, la crítica, la participación constructiva para superar los retos de la sociedad. Para ello recurre a un enfoque pedagógico y actividades académicas que permiten a sus estudiantes el desarrollo de destrezas específicas y la apropiación de valores consecuentes con ese compromiso. Buscan promover y facilitar el intercambio de experiencias pedagógicas innovadoras entre el personal académico y estudiantes del colegio con otras instituciones educativas de la región y del país, que favorezcan el análisis de las diferencias o semejanzas de las problemáticas educativas que enfrentan los diferentes contextos educativos. Entre los aspectos innovadores de este sistema educativo es que promueve los estudios de profundización en las áreas de pensamiento contemporáneo, Humanismo, educación, conocimiento, pensamiento crítico, sociedad, lengua y literatura, historia y sociedad e inglés de profundización. 
Palabras clave: Humanismo, educación, conocimiento, pensamiento crítico, sociedad.

\section{Abstract}

The system of humanistic secondary schools promotes the humanistic education of citizens with commitment in the analysis, the criticism, and the constructive participation to overcome challenges of the society. For this, it has recourse to a pedagogical approach and academic activities letting students to develop their specific skills and to acquire values consistent with this commitment. They try to promote and facilitate the exchange of pedagogical and innovative experiences among the academic staff and the secondary school students with other educational institutions of the region and of the country, in order to favor the analysis of differences or similarities of educational problems that different educational contexts face. One of the innovative aspects of this educational system is that it promotes in-depth studies of the areas of the contemporary thought, language and literature, history and society, and in-depth English.

Keywords: Humanism, education, knowledge, critical thinking, society.

1 n la década de los noventa, líderes de la academia de la Universidad Nacional (UNA) y del sector educativo visualizaron la necesidad de un nuevo sistema pedagógico para el país, desde la proclama de que "el humanismo promueve la realización de la persona y que para lograr esto, es necesario que el individuo se conozca en su calidad de sujeto libre, pensante y creador, que sea portador de valores, derechos, y deberes; y, a la vez, se sienta comprometido con la sociedad, en su realidad actual y en su transformación” (Colegio Humanístico Costarricense, 2009); la cual inspiró la propuesta de un grupo de docentes de la UNA, quienes plantearon una inquietud: ¿cómo democratizar el sistema educativo costarricense para estudiantes con intereses especiales en las letras y ciencias sociales, sin menoscabo del desarrollo de las ciencias exactas y naturales?

Como respuesta a esta interrogante surge la propuesta de crear una institución de educación diversificada, donde se potencien las Humanidades y la formación integral de sus estudiantes en un ambiente crítico ante los cambios políticos, económicos y culturales del mundo actual. De esta forma, la fundación del sistema de colegios humanísticos costarricenses se realiza mediante Decreto Ejecutivo 26436 del 16 de octubre de 1997 y se da la apertura del primer Colegio 
Humanístico Costarricense (CHC), con sede en el Campus Omar Dengo de la UNA, en febrero de 1998. Posteriormente y mediante acuerdo n. ${ }^{\circ}$ 312, publicado en la Gaceta N. ${ }^{\circ} 115$ del 17 de junio del 2003, se aprueba la creación del segundo Colegio Humanístico Costarricense Campus Coto, propiamente en la zona sur de nuestro país, el cual inicia sus funciones en febrero de 2004.

El sistema de colegios humanísticos busca promover y facilitar el intercambio de experiencias pedagógicas innovadoras entre personal académico y estudiantes con otras instituciones educativas de la región y del país, que permitan el análisis de las diferencias o semejanzas en las problemáticas educativas que enfrentan los diferentes contextos educativos. Dentro de lo innovador del sistema es que permite y contribuye con los estudios de profundización en las áreas del pensamiento contemporáneo, lengua y literatura, historia y sociedad e inglés que favorecen al estudiantado, en su marco de referencia, para ampliar criterios de discusiones acerca de las problemáticas actuales.

En el caso del CHC- Campus Coto, se reciben estudiantes de los cinco cantones de la zona sur-sur de nuestro país (Golfito, Osa, Corredores, Coto-Brus y Buenos Aires), y en el CHC - Campus Omar Dengo, ubicado en Heredia, se realiza un proceso de admisión que incluye a las siete provincias del país. Recibe, por lo tanto, estudiantes de todo el territorio nacional que muestren interés por una formación integral dentro de parámetros de alta calidad y desde una perspectiva humanística, sin que sean un obstáculo las condiciones socioeconómicas, familiares o de ubicación geográfica para su ingreso; incluso, como apoyo a estudiantes de escasos recursos económicos, se cuenta con un sistema de becas, para que estas razones no limiten sus aspiraciones de optar por una mejor formación.

Es importante señalar que el sistema de colegios humanísticos costarricenses, de la Universidad Nacional, tiene como propósito formar estudiantes de IV ciclo de educación diversificada, a través de una opción educativa dinámica que responda a las demandas de la cambiante sociedad contemporánea y las transformaciones capitales que tienen lugar en los procesos de generación y transmisión de conocimientos.

Este modelo educativo es una alternativa que le permite al estudiantado poder desarrollar su potencial humano, construir y fortalecer los valores que guiarán su vida, mediante cada una de las acciones que conducen al éxito a través de la 
excelencia académica, artística, cultural, deportiva y espiritual en las que se ve inmerso en los diversos entornos de los centros educativos.

El énfasis en la excelencia académica pretende no solo impactar en estos colegios, sino a través de él y de otras expresiones, permear al sistema educativo en su conjunto; así como promover determinadas áreas del conocimiento para responder a la vocación especial, de ciertos grupos estudiantiles, en áreas que satisfagan su desarrollo personal pleno y que resultan imprescindibles para impulsar un desarrollo nacional armónico.

Existe un vínculo directo con la UNA como casa de estudios superiores; esto posibilita el hecho de que el estudiantado desarrolle su formación en el campus universitario, en sus bibliotecas, en las clases de algunos profesores o profesoras de la UNA, trabajando en proyectos de investigación y extensión.

De manera complementaria y puntual, cabe cuestionarse por qué desde la Universidad Nacional se promueve la realización de un proyecto de extensión con la población estudiantil de los colegios humanísticos, instituciones de educación media, la respuesta a esta interrogante tiene varios asideros:

- En términos amplios es importante considerar que la Constitución Política de la República señala en el título VII relativo a la Educación y Cultura, Artículo 77: "La educación pública será organizada como un proceso integral correlacionado en sus diversos ciclos, desde la preescolar hasta la universitaria” (Constitución Política de la República de Costa Rica, 1949).

- En particular, la Universidad Nacional adquirió el compromiso con el Colegio Humanístico de Coto desde que este colegio quedó adscrito a la UNA (Convenio específico entre la UNA y el MEP para apoyar al Colegio Humanístico Costarricense, Campus Coto, 10-01-2005). Este está basado en el Decreto Ejecutivo N²6436 MEP de 1997, que establece la creación del Colegio Humanístico Costarricense Campus Omar Dengo. De esta forma se establece un convenio entre la UNA y el MEP para apoyar al sistema de colegios humanísticos costarricenses, en el cual se indica de tácita que tanto la UNA como el MEP son instituciones llamadas, por razón de su esencia, finalidad y objetivos, a establecer canales de comunicación que permitan el desarrollo conjunto de proyectos y actividades tendientes al mejoramiento cualitativo de la educación en el país. 
- La participación de la Universidad Nacional en la creación de los colegios humanísticos costarricenses se justifica en la responsabilidad histórica que esta institución ha adquirido, desde su fundación, con los sectores menos privilegiados de la sociedad costarricense (Plan Global Institucional, 20042011), entre ellos los pueblos indígenas. De manera concatenada, merecen especial atención, para los efectos de esta propuesta, los incisos del Plan Global Institucional que aluden al respeto a la diversidad inclusiva (C); a las oportunidades para los sectores más vulnerables (E); a la valoración positiva de la diversidad cultural $(\mathrm{H})$; y de manera más totalizadora, a una sociedad que haga efectivos los principios de equidad, justicia social, participación ciudadana y respeto pleno a los derechos humanos (I) (Plan Global Institucional, 2004-2011), principio este que se reitera de modo sustancial en la Misión de la Universidad.

Más de tres quinquenios han pasado desde que surge este modelo de educación preuniversitaria, con la clara convicción de estar cumpliendo con la misión de ofrecer un modelo comprometido con el desarrollo del talento y la apropiación de valores que promueven el pensamiento analítico, reflexivo, ético y trascendente; se han desarrollado varias generaciones graduadas con un enfoque caracterizado por la excelencia académica en el marco de una visión humanista, con énfasis en áreas del conocimiento que satisfacen el desarrollo personal pleno e impulsan el desarrollo nacional armónico; se trabaja con profesorado con experiencia y trayectoria universitaria; existe un vínculo directo con su casa de estudios superiores (UNA); se brinda una formación integral que promueve el desarrollo de la capacidad crítica, analítica y creativa; se propicia la sensibilidad social, artística y sus relaciones con la naturaleza; se busca la autonomía y la responsabilidad; se genera la solidaridad, el respeto y la tolerancia al facilitar el trabajo en equipo y considerar los intereses, así como las necesidades de sus estudiantes, con lo cual se reafirma el compromiso institucional que este modelo educativo asumió con la formación educativa del país.

\section{Origen del colegio}

El Colegio Humanístico Costarricense surge como una propuesta dirigida al Ministerio de Educación Pública con sello de la Universidad Nacional. Se visualiza como una respuesta ante las críticas surgidas por los cambios culturales impulsados, producto de la globalización de la economía. 
El 16 de octubre de 1997, a través del Decreto Ejecutivo N. ${ }^{\circ} 26436$ MEP, se inaugura la trayectoria del primer Colegio Humanístico Costarricense, como producto de la inspiración de un grupo de profesores de la Universidad Nacional, de las más diversas áreas académicas, coordinado por la doctora Sonia Marta Mora Escalante, con el fin de brindar un espacio a estudiantes de décimo y undécimo nivel con aptitudes destacadas en los campos de las ciencias sociales y las letras.

Los considerandos del Decreto que articulan los principios éticos, filosóficos y motivacionales del colegio son:

a. El Consejo Superior de Educación, al ejercer la dirección de la enseñanza oficial, conocer los proyectos de creación de nuevos tipos de colegios... deberá procurar una educación de excelente calidad y que responda a las demandas de la sociedad actual.

b. El dinamismo de los cambios de la sociedad contemporánea y las transformaciones capitales que tienen lugar en los procesos de generación y transmisión del conocimiento hacen más urgente que nunca la construcción de opciones educativas innovadoras. En especial resulta urgente la implementación de opciones que enfaticen la reflexión y formación de valores humanos fundamentales y que, así, respondan a los hondos desafíos éticos de la época actual.

c. El sistema educativo nacional debe ofrecer una modalidad educativa que coloca al ser humano, su pensamiento, su historia, su legado y su relación armónica con el mundo natural, en el centro de todo esfuerzo de conocimiento.

d. En Costa Rica existe un considerable grupo de estudiantes con destacadas destrezas en el campo de la expresión verbal, la filosofía, la historia y la crítica del pensamiento y la cultura. Dado que actualmente estos estudiantes no cuentan con una opción intensificadora que les permita desarrollar esas destrezas, se lesiona el sentido de auténtica justicia y de creación de oportunidades y se resta posibilidades al país de desarrollar sus potencialidades.

e. En este contexto los Colegios Humanísticos constituyen entonces un elemento reforzador de la democracia, en la medida en que los estudiantes de todos los sectores sociales podrán acceder a ella con base en su esfuerzo y con independencia de su capacidad económica. Consecuentemente, abrir una opción a los estudiantes con vocación para las ciencias sociales y las humanidades enriquece al sistema educativo nacional en su totalidad.

f. Por otra parte, estos colegios deberán tener vínculo directo con una Casa de 
Estudios Superiores (Universidad Estatal).

Los CHC más que una respuesta ante un vacío en el sistema educativo costarricense, el cual ya contaba con varias instituciones de corte científico, deportivo, artístico, ambiental; sin embargo, ninguno en el que la juventud pudiera desarrollar sus habilidades integralmente en los diversos saberes relacionados con el arte, las letras, las ciencias exactas y naturales. Son, entonces, el resultado del pensamiento crítico y reflexivo ante los retos impuestos por la sociedad contemporánea y un imperante cientificismo, como única alternativa educacional al sistema regular de enseñanza secundaria.

El propósito de hacer de los colegios humanísticos un lugar de prestigio se ha cumplido (a lo largo de 17 años en el campus Omar Dengo y 11 en el campus Coto), al amparo de su casa de estudios superiores, la Universidad Nacional, con la cual posee un vínculo directo por medio del Convenio UNA-MEP, fechado el 20 de noviembre de 1997. Este permite al estudiantado del colegio desarrollarse en el campus universitario, ambiente idóneo para la reflexión y la crítica, dos de sus pilares fundamentales, cuyo fin es la construcción de un mejor ser humano.

\section{La experiencia más reciente: $\mathrm{CHC}$-Campus Coto}

Dentro de los objetivos de creación del colegio esta apoyar el desarrollo de dicha institución en la región educativa de Coto, en el cantón de Corredores, distrito de Paso Canoas. Este se regirá por criterios de calidad y excelencia académica. Fortalecerá las experiencias educativas innovadoras en las asignaturas que conforman el plan de estudios en el nivel de la educación diversificada académica, con el propósito de favorecer el desarrollo máximo de las potencialidades del estudiantado, en el marco de una educación humanística.

Es importante señalar la búsqueda permanente y el desarrollo de poner en práctica un proceso de investigación pedagógica que permita una actualización permanente de su plan de estudios, de manera que sea más acorde con las necesidades y demandas que plantea la comunidad educativa y el contexto.

Otro aspecto significativo es que se busca un mejoramiento académico en la región como un mecanismo que contribuya a incrementar la calidad de vida de sus habitantes. 
En el convenio UNA-MEP se establece un apartado en donde se pide que se involucre a estudiantes de los colegios humanísticos en extensión e investigación, lo cual se desarrolla con mayor solvencia en el campus Omar Dengo; sin embargo, se presenta cierta limitación en el campus Coto, con la dificultad de que, en la UNA, campus Coto, al ser un campus relativamente joven, no se contaba con ese tipo de proyectos. De ahí que en el año 2014, la administración y demás personal se dio a la tarea de participar en la Convocatoria FUNDER (Fondo Universitario para el Desarrollo Regional) logrando que la Universidad Nacional brindara un apoyo adicional y aprobara el proyecto "Construyendo espacios de diálogo intercultural desde el Colegio Humanístico de Coto”, donde se involucran estudiantes del colegio con otras instituciones, sobre todo de secundaria, en territorios indígenas en torno a los derechos humanos.

El proyecto tiene el propósito de poner en práctica una propuesta que genera espacios para la construcción y fortalecimiento de relaciones interculturales respetuosas, tolerantes y solidarias, entre estudiantes no indígenas e indígenas de la región Brunca, en relación con los derechos humanos de los pueblos indígenas. En términos más amplios, el proyecto contribuye a traer el tema de los derechos humanos de pueblos indígenas al análisis y reflexión del Colegio Humanístico del Campus Coto y del cantón de Corredores.

En estos espacios, el alumnado del CHC y estudiantes indígenas tienen la oportunidad de trabajar de manera mancomunada en el campus Coto y en territorios indígenas. Así, comparten ideas, experiencias y conocimientos sobre los derechos humanos de pueblos indígenas, temática que se constituye en el eje transversal y referente constante de este proyecto.

Este acercamiento a los derechos humanos se alcanza a lo largo de distintos espacios organizados con este propósito: talleres, conversatorios, encuentros de socialización y giras pedagógicas. Dentro de estos espacios, el estudiantado genera productos tangibles como memorias fotográficas y ensayos, lo cual produce la construcción de productos no tangibles como el fortalecimiento del respeto ante la diversidad cultural y la contribución a las relaciones interculturales armoniosas y tolerantes.

Esta perspectiva ha sido sustentada e impulsada por instancias gubernamentales y organismos internacionales, entre ellos, el Programa de las Naciones Unidas para el Desarrollo (PNUD) adoptó el desarrollo humano sostenible desde 1990. 
También los pueblos indígenas en años recientes han planteado su contribución en este sentido, con la visión del desarrollo como "buen vivir o vivir bien”.

Esta iniciativa fue construida inicialmente entre el Colegio Humanístico de Coto y el Programa Asesoría en Investigación, Asistencia Técnica e Información Estratégica del Instituto de Estudios Sociales en Población(IDESPO), denominado a partir del 2014 como Programa estudios de población para la equidad con perspectiva de género y diversidad cultural.

El procedimiento metodológico empezó a construirse a partir del análisis de las experiencias del equipo académico proponente $\mathrm{y}$, sobre todo, desde los valiosos planteamientos hechos por estudiantes del colegio humanístico durante conversatorios sostenidos en el campus Coto. De estos encuentros destacaron las siguientes sugerencias:

- Que el proyecto se constituya en una alternativa para su práctica de trabajo comunal, requisito esencial establecido por el Ministerio de Educación para el ciclo diversificado.

- Que el proceso de aprendizaje sobre los derechos humanos de pueblos indígenas se lleve a cabo de una manera dinámica y amena, y que se abran espacios para compartir con personas indígenas tanto en el campus, como en sus territorios.

- Que en este proceso se vislumbre la necesidad de elaborar una propuesta que incluya aportes metodológicos provenientes de la educación formal, y también prácticas propias de la educación informal sustentada por los pueblos indígenas.

Desde su fundación, el CHC se ha caracterizado por una experiencia muy exitosa, ya que ha logrado complementar con resultados altamente positivos en las pruebas nacionales, en una región deprimida en todos los sentidos. Algunas breves consideraciones sobre los logros del colegio humanístico desde su fundación hasta este periodo son:

- Por las características propias de la región, es muy poca la cantidad de estudiantes que logran ingresar a las universidades públicas; sin embargo, esta institución, por efecto de su formación integral, ha podido ir creando una élite de bachilleres capaces de enfrentar con éxito la educación superior. 
- El estudiantado del colegio se ha caracterizado, desde sus inicios, por situarse entre los mejores colegios públicos de nuestro país, ya que siempre ha obtenido $100 \%$ en las promociones de Bachillerato Nacional.

- En el año 2006 ocupó el primer lugar a nivel nacional en las pruebas de Bachillerato, por encima de todos los colegios públicos y privados del país.

- A pesar de que su enfoque es más dirigido hacia las ciencias sociales, sus estudiantes participan activamente en las olimpiadas de matemáticas, química y biología y física; así como en los cursos de Precálculo y Química General de la UNA.

- En las actividades a nivel regional el colegio siempre ocupa lugares de privilegio, llámese Festival de inglés, Saber matemático, FEA, entre otros.

- El estudiantado que egresa se incorpora con éxito a las universidades públicas (UNA, TEC, UCR).

Adicional a esto, quizás uno de los más importantes logros institucionales es ir creando un grupo de profesionales con arraigo en la región y compromiso con el desarrollo de la zona y del país.

\section{Naturaleza y filosofía del sistema de $\mathrm{CHC}$}

El sistema de colegios humanísticos costarricenses fomenta la formación humanista de una ciudadanía comprometida con el análisis, la crítica, la participación constructiva para superar los retos de la sociedad. Para ello, recurre a un enfoque pedagógico y actividades académicas que permiten, a sus estudiantes, el desarrollo de destrezas específicas y la apropiación de valores consecuentes con ese compromiso.

Su carácter preuniversitario facilita el acceso a conocimientos, el desarrollo de habilidades intelectuales y de convivencia académica que garanticen la rápida y oportuna inserción de sus graduados y graduadas en los estudios superiores a través del vínculo estrecho entre sus docentes y el quehacer académico de la Universidad Nacional, así como el acceso a las bibliotecas, los laboratorios y espacios de interacción cultural propios de la UNA, sin detrimento de los aportes y proyectos propios del cada CHC. 
El quehacer institucional se basa en el humanismo, cuyo objetivo es desarrollar al máximo las potencialidades de los individuos a través de la libertad creativa, transformadora y autónoma, orientada por la búsqueda de la sabiduría y el bien común. Esta labor propicia la construcción de un individuo solidario, empático, propositivo y comprometido en el desarrollo social justo y equitativo, por ello, sus estudiantes muestran interés en los debates de ideas, observan el mundo, aman la libertad y transforman su presente a través del trabajo.

Estos centros educativos incorporan las humanidades en su currículo, porque ayudan a construir el entendimiento con el fin de comprender el accionar humano para transitar de pasadas a nuevas formas de vida y comportamientos sociales. De este modo, filosofía, historia, literatura, arte y aprendizaje de idiomas, como espacios académicos, contribuyen, con especial énfasis, al debate y apropiación de valores humanistas, mientras que, las materias básicas están destinadas a garantizar aprendizajes de calidad desde una perspectiva humanística, al fomentar el compromiso social y ético en cualquier profesión que desempeñe quien egresa de sus aulas.

Desde la visión del CHC, el concepto de humanismo es desarrollado como una forma de vida que implica un enfoque filosófico centrado en el individuo, con el fin de propiciar el desarrollo físico, psicológico, social y espiritual. Con rigurosidad académica se procura el crecimiento del ser humano en armonía consigo, con las otras personas y con su entorno, tomando en cuenta su diversidad, sus espacios y su momento histórico. En su dimensión pedagógica, busca educar para la paz, los derechos humanos, la democracia y la sana convivencia formadora de criterios. Además, se fortalece en los pilares que rigen el quehacer educativo institucional, los cuales son:

a. Procurar una educación de excelente calidad que responda a las demandas de la sociedad actual.

b. Implementar opciones que enfaticen el pensamiento reflexivo y la formación de valores humanos fundamentales como respuesta a los desafíos éticos de la época actual.

c. Colocar al ser humano, su pensamiento, su historia, su legado y su relación armónica con el mundo natural, en el centro de todo esfuerzo y conocimiento.

d. Brindar oportunidades a estudiantes con destacadas destrezas en el campo de la expresión verbal, la filosofía, la historia, la crítica del pensamiento y la cultura. 
e. Los colegios humanísticos son un elemento reforzador de la democracia: es para todo estudiante con el perfil indicado independientemente de su capacidad económica.

f. Los colegios humanísticos deberán tener vínculo directo con una casa de estudios superiores estatal.

Desde esta perspectiva, el humanismo deja de ser un refugio filosófico donde se discuten problemas, y se convierte en un espacio para repensar, releer y reconstruir la sociedad, formando una persona ética, por medio de un acto libre y racional. Por eso, la educación humanística favorece el conocimiento de los diversos puntos de vista de la realidad y las interpretaciones ideológicas, los debates sobre las cuestiones y la dinámica social a partir de la observación de la vida cotidiana para así examinarla, abrir espacios para asumir valores, así como tomar conciencia de nuestros roles individuales y colectivos.

\subsection{Objetivos académicos}

Los objetivos propuestos a nivel institucional están basados en el desarrollo de acciones para la enseñanza y el aprendizaje, con un alto grado de compromiso social. Por otra parte, proponen mejorarlos niveles de aprendizaje de las materias correspondientes al ciclo diversificado. Además, generar espacios para la profundización de conocimientos y el desarrollo de destrezas y actitudes en el campo de las humanidades. De esta forma se aumentan los niveles de compromiso del estudiantado y del personal académico con la excelencia, en acciones que permiten una formación integral, interdisciplinaria, participativa, comprometida con la sociedad y el ambiente; se promueve que el alumnado se distingan por su capacidad de diálogo, de autorreflexión, de crítica; con sensibilidad social; gusto por aprender y aplicar lo aprendido; creador de respuestas concretas ante los problemas, y con compromiso en la construcción de un mundo mejor para todas las personas.

\subsection{Enfoque pedagógico}

El colegio humanístico percibe a sus estudiantes como aprendices a partir de experiencias gratificantes que fomentan la confianza en la naturaleza humana, sus estilos de aprendizaje, en el conocimiento aprendido, la incorporación de sus opiniones y sentimientos. 
Esta institución contribuye con sus colegiales para que construyan conceptos propios de sí y del mundo, a partir del acercamiento a la realidad, para comprometerse con la vida, el colectivo social, el trabajo y los afectos. Las metodologías utilizadas muestran confianza en las capacidades e intereses de las personas por aprender, resolver problemas y construir soluciones con el fin de que se apropien de valores positivos para la sana convivencia.

\subsection{Principios orientadores del modelo pedagógico}

Entre los principios fundamentales que rigen la formación académica del sistema de colegios humanísticos, se destacan:

- Concibe la acción de aprender como un proceso constructivo, resultado del trabajo en equipo.

- Aborda la investigación seria y rigurosa como una forma para construir conocimiento.

- Impulsa el diálogo y la discusión como formas permanentes para construir la verdad socialmente.

- Respeta las diferentes formas de aprender.

- Fomenta la interdisciplinariedad entre los diferentes cursos.

- Aspira a evaluar los procesos de aprendizaje antes que los resultados.

En conclusión, los colegios humanísticos costarricenses fueron fundados como una forma de democratizar el sistema educativo costarricense, con el fin de ofrecer una alternativa diferente, especial y específica, que enfatizara el área de letras y ciencias sociales, sin olvidar el desarrollo de las ciencias exactas y naturales. Constituye, así, una alternativa dirigida a jóvenes con una visión diferente de estudio, con compromiso e identificación con el quehacer de la academia y la labor intelectual.

Además de la formación académica, se ha propiciado un modelo educativo centrado en la persona, en sus cualidades y sus experiencias, el cual estimula al estudiantado a conocerse y a definir un proyecto de vida instaurado en la visión de la educación humanística.

En esta propuesta educativa, ha sido necesario identificar los talentos en las distintas áreas, para dar sentido y significado al aprendizaje, con el fin de propiciar el desarrollo de una visión personal del mundo, pero que responda a la demanda 
de un contexto exigente y versátil, por lo cual se cultiva la reflexión, se fomentan los valores y el compromiso con el ser humano y se promueve en el alumnado la capacidad de transformar, mejorar su entorno, procurar la excelencia en su esencia desde un enfoque humanista del conocimiento.

Esta tarea que ha sido asumida por quienes, de diversas maneras, han desarrollado identificación con este centro educativo, generando nuevas destrezas en la búsqueda del saber, desarrollando el potencial y la capacidad creativa a partir de un modelo basado en la visión y misión de la Universidad Nacional, y compartiendo su filosofía como una opción para toda la población.

En poco más de 17 años, los objetivos y metas con que se concibió este proyecto se han ido consolidando paso a paso, gracias al esfuerzo del personal docenteadministrativo, a la confianza de los padres y madres de familia en este modelo pedagógico, a la dedicación de sus miembros de Juntas Administrativas, así como a la entrega y voluntad de la generación de jóvenes que han sido y son su razón de ser; quienes, con el apoyo de las autoridades de la UNA y del MEP han demostrado que, con los estímulos necesarios y creando los espacios adecuados, se pueden potencializar al máximo las habilidades de la juventud.

\section{Referencias}

Colegio Humanístico Costarricense. (1999-2014). Actas de Juntas Administrativas del Colegio Humanístico Costarricense. Archivos del Colegio Humanístico Costarricense 1997-2014. San José, Costa Rica.

Colegio Humanístico Costarricense. (Septiembre del 2009). Reseña histórica Colegio Humanístico Costarricense X Aniversario de Creación.

Programa de las Naciones Unidas para el Desarrollo. (1990). Informe sobre Desarrollo Humano.

República de Costa Rica. (16 de octubre de 1997). Decreto Ejecutivo 26439. San José, Costa Rica.

República de Costa Rica. (2010). Constitución Política de la República de Costa Rica. San José, Costa Rica: Publicaciones Jurídicas. 
Universidad Nacional y Colegio Humanístico Costarricense. (2003). Convenio entre la Universidad Nacional y el Colegio Humanístico Costarricense para el Reconocimiento de los 12 créditos de humanidades.

Universidad Nacional y Colegio Humanístico Costarricense. (2007). Convenio entre la Universidad Nacional y el Colegio Humanístico Costarricense para el Reconocimiento de los 12 créditos de humanidades. 